\title{
Towards an atomic-scale understanding of crystal growth in solution
}

\author{
Elias Vlieg, Menno Deij, Daniel Kaminski, Hugo Meekes and \\ Willem van Enckevort
}

\author{
Received 19th December 2006, Accepted 5th February 2007 \\ First published as an Advance Article on the web 8th May 2007 \\ DOI: $10.1039 / \mathbf{b 6 1 8 5 6 6 p}$
}

\begin{abstract}
Our understanding of crystal growth continues to increase thanks to progress in theoretical models, computer simulations and experimental techniques. A discussion of the state-of-the-art in morphology prediction and of the determination of the solid-liquid interface structure using X-ray diffraction shows, however, that there is still a large gap between experiment and theory. We expect that computer modelling, in the form of both Molecular Dynamics simulations and first-principle calculations, will play a crucial role in filling this gap.
\end{abstract}

\section{Introduction}

Whether one can claim to understand crystal growth depends strongly on one's perspective. At a simple level, crystal growth in solution can be understood to be the result of the supersaturation in a solution. Also in industrial crystallization a relatively simple power law may be adequate to describe the average size of a growing crystal population in time. However, if one aims at a fundamental understanding at an atomic level, many questions remain concerning the various mechanisms in the growth process. We will here discuss recent progress in this understanding as a result of theoretical and computational methods and modern experimental techniques. This will show that the gap between the quite detailed experimental information that is becoming available from realistic growth systems and the more simplified picture typically employed in computational and theoretical models, though getting smaller, is still large. Closing this gap requires a major effort in computer modelling and is an important challenge for the future.

Fig. 1 schematically depicts a crystal in a solution with a chemical potential that is higher than that of the crystal. We will not discuss nucleation here, since this is even less understood and is a research field of its own. The growth can be divided into two parts: (1) the transport of the growth units towards the crystalsolution interface and (2) the incorporation into the crystal at the surface. The description of mass transport does usually not require an atomic-scale description and a continuum model is therefore appropriate. Mass transport can be quite complex, involving the interplay of convection, diffusion and, possibly, forced flow, but overall this is quite well understood. Software is available to model this under realistic conditions.

IMM Solid State Chemistry, Radboud University Nijmegen, Toernooiveld 1, 6525 ED, Nijmegen, The Netherlands 


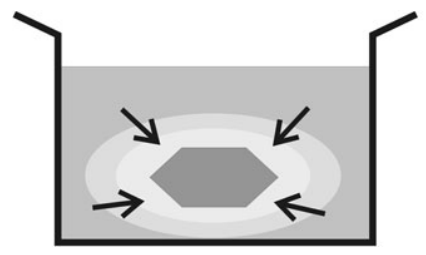

View Article Online

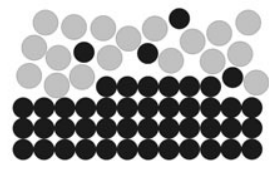

Fig. 1 A schematic of crystal growth in solution. Mass transport in the solution can be described on a macroscopic scale. The actual incorporation of growth units at the solid-liquid interface requires an atomic-scale description.

The second step, involving the interface is much less understood and does require a description at the atomic level. A growth unit (which may contain a shell of water/ solution molecules) has to diffuse through the solution layers near the crystal surface. These quasi-liquid layers provide kinetic barriers of a different kind than the bulk liquid, because of their interaction with the crystal surface. The liquid will be more ordered than the bulk liquid and may even be chemisorbed to the crystal. If the growth unit contains a water/solution shell, this needs to be partly removed before contact with the crystal surface can be made. The crystal surface itself may also deviate from its bulk structure and thus present a different bonding geometry than expected. Kinks and steps are the most important growth sites. Growth units may either be incorporated at these sites directly from the solution or first diffuse over the surface. The step and kink density depend on the step and kink free energy, which will be influenced by the solvent used. This description is still simplified, because parameters like temperature, electrochemical potential, defect concentration and impurities/additives can all play a role. Even when ignoring these parameters, a full description of solution growth is a formidable task.

Let us now try to evaluate how well we understand these atomic-scale processes. In this context it is useful to make a distinction in system complexity and system size. Simple model systems are of course better understood than growth under realistic conditions. Fig. 2 provides a scheme with complexity and size as parameters. The essential ingredients for understanding crystal growth are contained in the seminal paper by Burton, Cabrera and Frank from 1952. ${ }^{1}$ This has formed the basis of a host of subsequent theoretical papers, including computer simulations. The focus of these

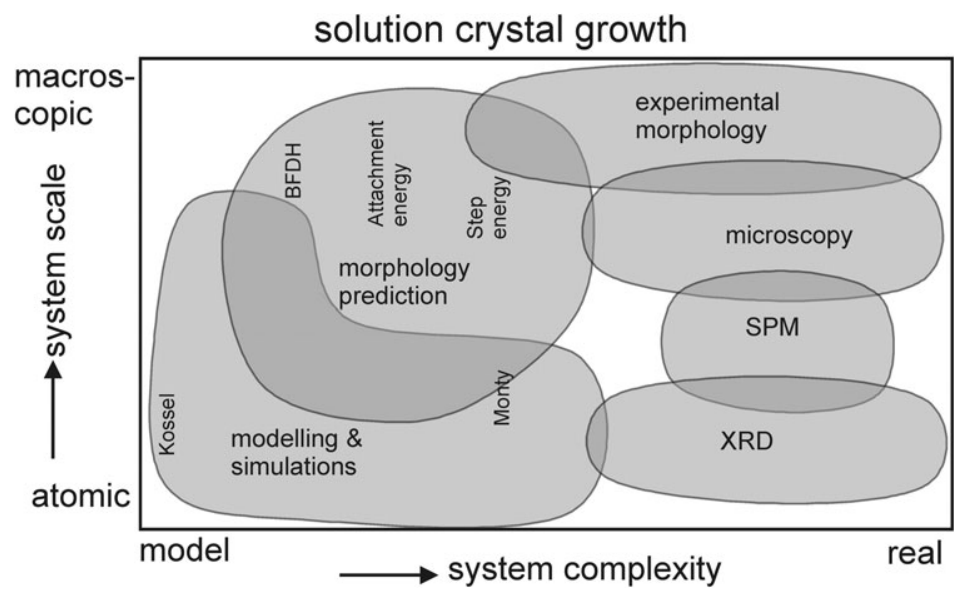

Fig. 2 A diagram showing the realms of experimental, theoretical and computational methods for investigating crystal growth in solution and using system complexity and system scale as parameters. Experimental methods always deal with real systems, while modelling has to simplify reality. A number of specific models that are mentioned in the text are indicated by vertical text. 
papers, however, was on simple model systems, in particular on the Koss@iewystrible Online The Kossel crystal has been essential in elucidating many aspects of crystal growth and continues to play this role. ${ }^{3}$ At this simple model level, no specific features occur at larger length scales and thus the left side of our complexity-scale diagram is fully covered by theory and computer simulations. More realistic systems pose a greater challenge. Several model systems with more complexity or different bonding than the Kossel case have been investigated. 'Non-Kossel' models containing more than one growth unit per unit cell are found to be quite challenging. ${ }^{4,5}$

More realism is also aimed for in Molecular Dynamics computer simulations in which model potentials describe the interactions between molecules. Such simulations can provide not only an approximate description of the solid-liquid interface structure, but also estimate kinetic barriers as was demonstrated in the pioneering work of Liu et al. on the urea-water interface. ${ }^{6}$ More simulations of this type have since been done, but computer power remains a bottle neck and thus the system size needs to be small. Even more demanding are full quantum-chemical calculations of the structure of a solid-liquid interface using e.g. density-functional theory and such results are starting to appear only now. ${ }^{7}$ All in all it can be stated that computer modelling is slowly gaining territory in the complexity direction, but only on small systems and with several parameters from real systems still left out.

While having overlap with computer modelling and simulations, the realm of morphology prediction can be treated as somewhat separate and has a different goal: predicting the shape of a growing crystal. This field lies in the middle section of our diagram. Progress in this area will be discussed in the next section.

Experimental observations of crystal growth are always on 'real' systems, but of course one can study model systems that are chosen for their simplicity. Even the simplest experimental model system, however, will be far more complex than the Kossel crystal. Thus all experimental observations are located at the right side of our complexity-scale diagram. Crystal morphology on a macroscopic scale has been studied for centuries and a lot of phenomenological knowledge is available about the influence of parameters like temperature, solution and impurities on the morpho$\operatorname{logy} .{ }^{8,9}$ Visual inspection, or, more quantitatively, an optical goniometer, is sufficient at this level of detail. Optical microscopy brings the length scale down by several orders of magnitude and electron microscopy allows even smaller details to be visualized. At the $\mu \mathrm{m}$ length scale, the crystal morphology is often 'surprisingly' similar to that at the macroscopic scale. For a fundamental understanding, it is therefore more relevant that microscopy can reveal, under favourable conditions, the growth mechanisms (spirals, 2D nucleation) and the shape of surface steps. Optical methods based on phase-shift interferometry have proven to be very powerful and continue to be refined for crystal growth applications. ${ }^{10}$

When scanning-probe microscopy (SPM) became available, it revolutionized several branches in science and crystal growth was no exception. The increased resolution enables the visualization of local structure in great detail, including step fluctuations and impurities. SPM experiments can be performed in situ, although real growth is often too fast for the technique and thus fairly low driving forces are needed. At the lower-right corner of our diagram we finally find X-ray diffraction, because this technique can provide information on the interface structure with the highest structural resolution. By its very nature, diffraction always provides an average picture and thus microscopy (local, moderate resolution) and diffraction (global, high resolution) provide information that is complementary in an ideal way. Recent progress in X-ray diffraction will be discussed in a separate section. Note that Fig. 2 also illustrates the well-known fact that a full experimental understanding requires the information from several techniques.

The next two sections describe in more detail recent progress in our group on morphology prediction and X-ray diffraction. This will show that despite this progress, there is still a long way to go before theories and crystal growth include all parameters that are experimentally known to be important. 
Here we discuss our recent progress in morphology prediction, i.e., in theories that aim to predict the shape of a growing crystal. This is an area lying between the detailed simulations of model systems and the world of real crystals. Predicting the morphology requires predicting the growth velocity of all relevant faces $(h k l)$, from which the morphology is derived using a kinetic Wulff plot. ${ }^{11}$ Theories for morphology prediction are based on the bulk crystallographic structure of a material and are thus more complex than e.g. the Kossel system. Using pragmatic simplifications, the theories are frequently applied in industrially-relevant situations.

The oldest theory was developed in several steps by Bravais, Friedel, Donnay and Harker some 100 years ago. ${ }^{12-14}$ This BFDH-theory ignores all details and simply states that the growth velocity of a facet is inversely proportional to the lattice spacing $d_{h k l}$. This 'explains' that facets on real crystals have typically low values for the Miller indices $(h k l)$. The BFDH theory works quite well for isotropic crystal structures, e.g., metals, but fails when the crystal structure becomes more complex. The bulk crystallographic structure was fully included in the Hartman-Perdok theory from the 1950s. ${ }^{15}$ This theory uses all the important bonds between the different growth units in the crystal. The essential structure of a crystal is represented in a crystal graph, showing the bonds between the various growth units, see Fig. $3 .^{16}$ In specific directions, one can construct so-called periodic-bond chains (PBC's) of growth units connected by bonds. A connected net is a $2 \mathrm{D}$ network consisting of two (or more) non-parallel, interconnected periodic bond chains. A stable crystal face, called a flat or F-face, will be parallel to such a connected net. The morphology of a crystal will mainly consist of these F-faces. The morphological importance of an

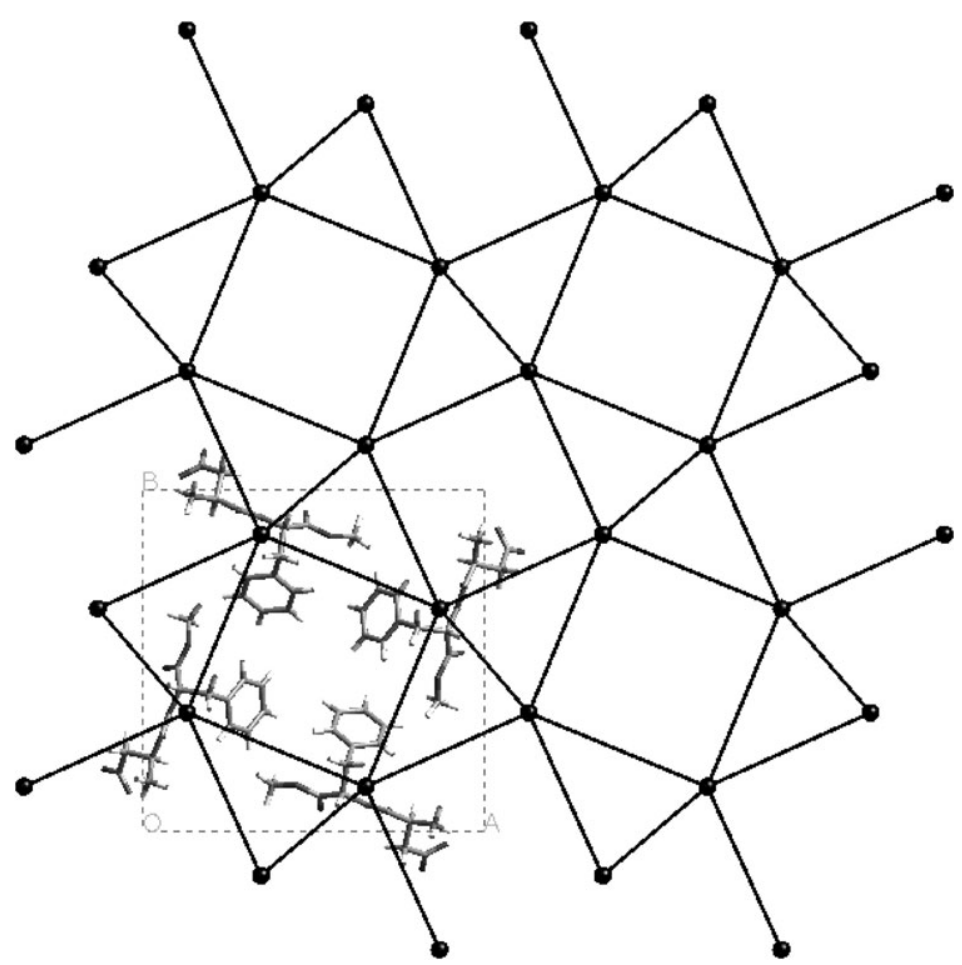

Fig. 3 2D projection of a crystal graph. The lower-left corner shows a full crystal structure (of aspartame). In a crystal graph, each growth unit is replaced by a node and the bonds between growth units are represented by an effective bond. The nodes plus bonds form the crystal graph. 
F-face in the Hartman-Perdok theory is derived from its attachment enerdfyięweArtible Online energy released when a complete layer with this orientation is crystallised. ${ }^{17}$ The growth rate is assumed to be proportional to the attachment energy. A software module called Facelift was developed that finds all possible connected nets in a given crystal graph and that can subsequently calculate the corresponding attachment energies. ${ }^{18}$ Using this, it is straightforward to calculate the attachment-energy morphology. For complex crystal structures, the number of $F$ faces can be very large, but typically only a small set of the most stable ones determine the morphology.

While highly successful, the Hartman-Perdok theory has been found to fail in several instances, typically involving structures with a low step energy. ${ }^{18}$ Since steps are well-known to be the dominant factor in crystal growth, it is not surprising that the classic Hartman-Perdok approach should fail, because only the attachment energy is considered. It is thus logical to develop a theory based on step energies and that is precisely what we have achieved recently.

Such a theory requires the determination of the energy of a single step and this is non-trivial except for simple model systems. The energy of a step can be defined as the difference in energy of a terrace with the step and that without the step. For the simple crystal structure shown in Fig. 4a, one can immediately see that this is well defined and that the step energy is that of a single bond. For more complex crystal structures, however, this is not so simple. Fig. $4 \mathrm{~b}$ shows a crystal model with two different atoms and two different bond strengths. When making a single step on such a surface, the top and bottom terrace are no longer vertically aligned, even though they are crystallographically equivalent. The result of subtracting the step-free terrace energy from that with the step, now depends on where one stops counting

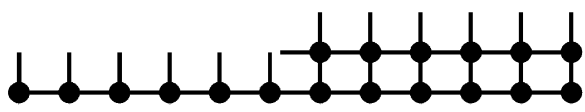

(a)
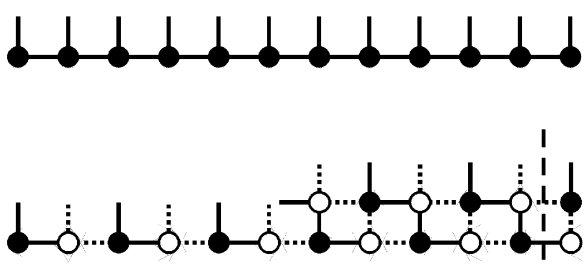

(b)
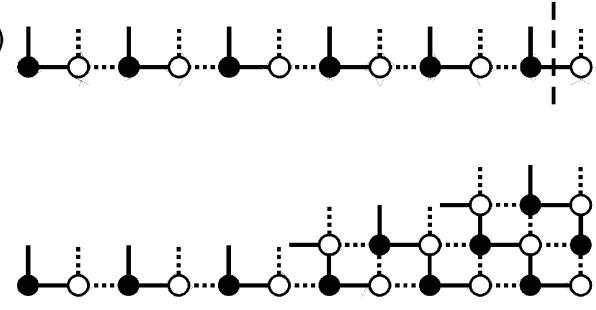

(c)

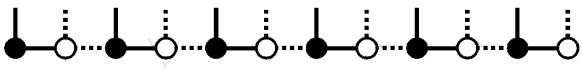

Fig. 4 Models for a stepped surface, projected along the step, together with a flat reference surface. The step energy is defined as the energy of the stepped surface minus that of the flat surface. (a) Kossel model. (b) Model with two different atoms and two different bonds. The calculated energy difference with respect to the flat surface depends on the boundary where one stops counting. (c) Same model as in (b), but now with two steps. This removes the ambiguity in subtracting the reference surface. 
behind the step. Using the entire Fig. 4b, the energy difference is found to beithaAroifce Online single dashed bond. However, when counting until the vertical dashed line, the result is a single solid bond. The ambiguity in this particular example can be resolved using the scheme in Fig. 4c. When taking two steps, the top-most terrace is again vertically aligned with the bottom one and subtracting the flat terrace is unambiguous. It is always possible to make the two steps identical as has already been done in Fig. 4c. The total energy of the two steps is found to be the sum of a solid and a dashed bond and thus the energy of a single step is half this value. The same principle can be applied to any crystal structure, but is more easily done by using a geometrical construction using a single step only. ${ }^{19}$ Our method thus disagrees with the notion that only the sum energy of two opposite steps is a well-defined quantity. ${ }^{20}$ Of course the absolute energy of a step does not exist, since it has to be determined with respect to a reference. For crystal growth, the most convenient reference is the flat surface. A further refinement would be to use the step free energy, because that is the real quantity of interest. We will not do that and use the step energy as a measure of the step free energy.

The possibility to determine the energy of a single step has also consequences for the estimate of surface roughening. If the step (free) energy of a crystal surface is zero, the surface will be rough. On a macroscopic scale, it will grow so fast that in most cases it disappears from the growth morphology. The condition for a surface to be flat, i.e. not rough, is usually given as:

$$
E_{\text {step }, \text { up }}+E_{\text {step, down }}>0,
$$

because a step up requires also a step down. ${ }^{21}$ According to this relation, even if the energy of the step up is negative, the sum of the energies can still be positive and the surface can still be flat. This is, however, not generally true. Using the single step energy as defined above, it is easy to give an example of a system that is thermally rough while still satisfying condition (1). Assume that the sum of two opposite steps is positive and that one of the step energies is negative. If the surface has three-fold symmetry, like on the $\{111\}$ surface of cubic crystals, then one can make a triangular island of which all sides have negative step energy. The opposite step with positive energy is not involved in constructing such an island. Generating such islands thus costs no energy and this surface will be rough. A more general relation for a surface to be flat is therefore:

$$
\oint E_{\text {step }}(\boldsymbol{u}) \mathrm{d} \boldsymbol{u}>0
$$

where $\boldsymbol{u}$ denotes the step direction of a 2D nucleus with equilibrium shape. Using a Wulff plot to find the island with the lowest energy, one can use eqn (2) to determine whether a surface will be rough or flat. In order to use this as a criterion for thermal roughening, one should of course use the surface free energy.

We have developed a computer code called STEPLIFT that can determine all possible steps on a surface and their energy. ${ }^{22}$ The procedure starts where the connected net determination of FACELIFT stops. Steps are constructed by the combination of the connected nets of two non-parallel F-faces, one representing the step terraces, the other the step edge. The structure of a specific step can be viewed in STEPLIFT, but the large number of possible steps makes an automated evaluation highly desirable. This is done by determining the broken bond step energy which, as stated before, largely determines the growth properties of a face. With many possible steps, each in principle with a different energy, we need to specify what is meant by 'THE' step energy. In order to arrive at a step energy value that is characteristic for a specific orientation, we use the average step energy of a $2 \mathrm{D}$ island with the lowest edge energy, i.e., of a 2D island with equilibrium shape. Such an island can be constructed from all calculated step energies using the 2D Wulff construction. We will denote this average step energy by $E_{\text {step, } h k l}$. 
With the step energy well-defined, we next need to relate this to tWeegromith Online velocity of each face. One very simple choice is to mimic the use of the attachment energy in the Hartman-Perdok theory and state that the growth rate is inversely proportional to the step energy. We can do much better, however, since we can use the more precise expressions from crystal growth theory. The growth velocity depends on the growth mechanism, i.e., whether growth proceeds through 2D nucleation or spiral growth. (In the case of rough growth the specific facet usually disappears from the growth morphology.) We will limit ourselves here to 2D nucleation, for which the following expression holds: ${ }^{23}$

$$
R_{h k l} \propto \beta_{\text {step }, h k l}\left(\frac{\Delta \mu}{k T}\right)^{5 / 6} \exp \left(\frac{-V E_{\text {step }, h k l}^{2}}{3 k T d_{h k l} \Delta \mu}\right),
$$

with $\beta_{\text {step }, h k l}$ the step kinetic coefficient, $\Delta \mu$ the driving force, $V$ the volume of a growth unit and $d_{h k l}$ the step height. If we assume that $\beta_{\text {step, } h k l}$ is approximately constant, i.e., its variations are small compared to the factors in the exponent, we have an expression for the relative growth rate of which all parameters are known. Compared with the earlier theories for morphology prediction, we even have a model that includes the driving force and the temperature. Fig. 5 summarizes the various steps to arrive at a morphology using the step energy model.

We give here one example of the application of the step energy model, for the morphology of a polymorph of venlafaxine ${ }^{\mathbb{R}}$, a pharmaceutical compound that works as an anti-depressant. The experimental morphology is shown in Fig. 6a. From the crystal structure ${ }^{24}$ the bond energies can be calculated using the Dreiding force field. For this case, the energies are scaled to yield the correct dissolution enthalpy in heptane, the actual growth solution. The crystal graph contains seven different bonds. After finding the various connected net orientations using FACELIFT, the morphology was calculated using both the attachment energy and the step energy model. Table 1 lists the corresponding energies for the orientations that are most relevant for the present discussion.

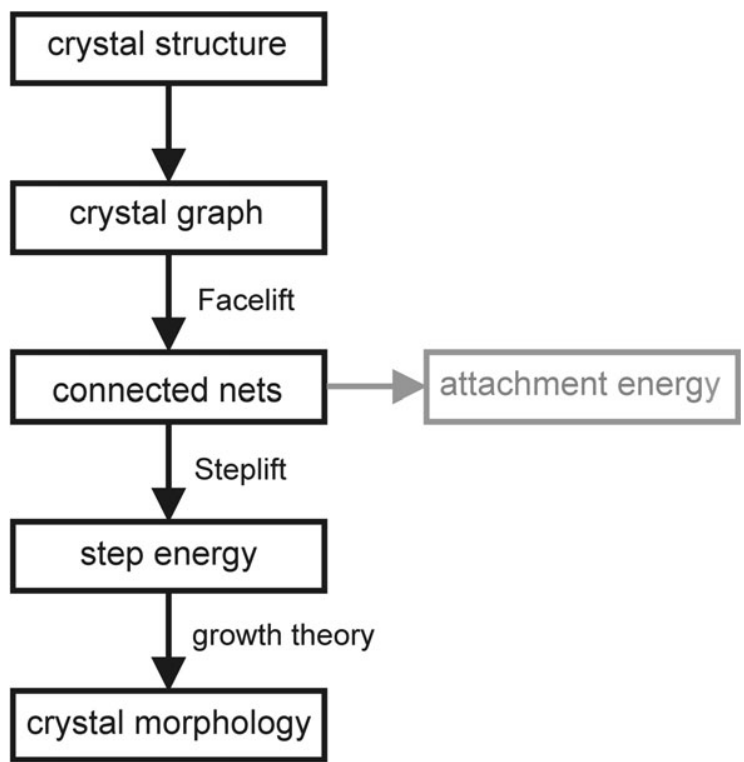

Fig. 5 The various steps in the determination of the step-energy morphology. The HartmanPerdok theory has the same starting point, but uses the connected nets to calculate the attachment energy as a measure for the morphological importance. 
(a)

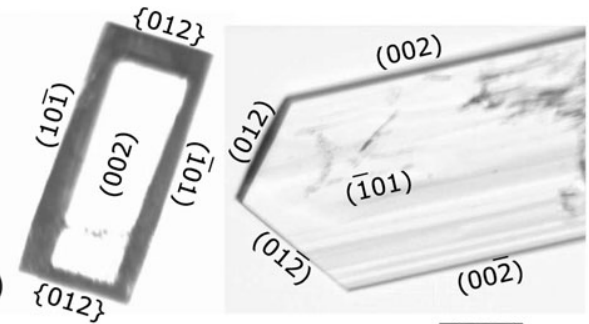

View Article Online

(b)
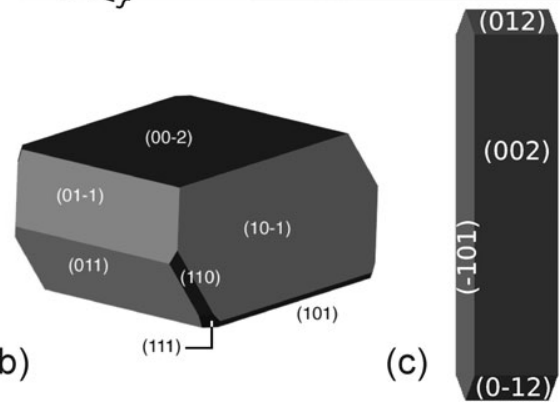

Fig. 6 The morphology of a specific polymorph of venlafaxine ${ }^{\circledR}$. (a) The experimental, (b) attachment energy and (c) step energy morphology. The step energy morphology agrees much better with the experiment than the attachment energy.

Fig. $6 \mathrm{~b}$ shows that the attachment energy morphology is quite different from the experimental one. The $\{01 \overline{2}\}$ orientation is observed but not predicted, while the $\{111\},\{110\},\{101\}$ and $\{011\}$ orientations are predicted but not observed. Furthermore, the $\{\overline{1} 01\}$ facet is less prominent than predicted. Fig. $6 \mathrm{c}$ shows the step energy morphology for $\Delta \mu / k T=4$. This agrees much better with the experiment: the three experimental facets are found and the shape agrees also quite well. Table 1 shows the characteristic difference between the two methods for the $\{01 \overline{1}\}$ facet. The attachment energy in this direction is small and thus this orientation is predicted to be important in the morphology. This facet, however, is found to be a case with very low step energy and thus the step energy method correctly predicts this facet to be absent. This is a clear case where the usual reciprocal relationship between attachment and step energies fails. ${ }^{17}$ The same is true for needle-shaped morphologies, where the step energy in the needle direction is found to be close to zero. ${ }^{25}$ For all these cases, the step energy method predicts a vastly improved morphology.

The choice for the value used for the driving force in the step energy prediction is not obvious. For venlafaxine ${ }^{\mathbb{R}}$ a value of $\Delta \mu / k T=4$ gives good agreement with experiment and lowering this value gives a more elongated shape, again in agreement with experiment. We find, however, that the theoretical values of the driving force required for the calculations are typically much higher than the experimental ones.

Table 1 The connected net orientations of venlafaxine ${ }^{\circledR}$ with the lowest attachment energies and the highest average step energies

\begin{tabular}{lll}
\hline$(h k l)$ & $E_{\text {att }} / \mathrm{kcal} \mathrm{mol}^{-1}$ & $E_{\text {step }} / \mathrm{cal} \mathrm{mol}^{-1} \AA^{-1}$ \\
\hline$\{002\}$ & -9.52 & 798.9 \\
$\{\overline{1} 01\}$ & -14.82 & 452.9 \\
$\{01 \overline{2}\}$ & -19.17 & 302.2 \\
$\{11\}$ & -25.03 & 150.2 \\
$\{110\}$ & -24.22 & 91.0 \\
$\{101\}$ & -20.01 & 73.3 \\
$\{01 \overline{1}\}$ & -19.04 & 25.2 \\
\hline
\end{tabular}


The origin of this discrepancy is not clear, but could be due to a spirtile wrontere Online mechanism for the real crystal. This needs further investigation.

\section{Atomic-scale structure at the solid-liquid interface}

Next we discuss experiments on the structure of the growth interface. Most models for crystal growth assume that the crystal surface has the same structure as the bulk. Several experiments performed in the last few years have shown that this is often not true. Two main techniques are available to determine the in situ structure of solidliquid interfaces: scanning-probe microscopy (SPM) and X-ray diffraction. SPM, often in the form of atomic-force microscopy (AFM), has (literally) provided a much better picture of the structure and role of steps. This has been highly relevant for a better understanding of crystal growth. ${ }^{26,27}$ For the determination of the atomicscale structure, however, X-ray diffraction (XRD) is the most suitable technique. ${ }^{28}$ $\mathrm{XRD}$ is widely used for the structure determination of crystal surfaces. ${ }^{29,30}$ Initially this was mainly carried out in a vacuum, but the large penetrating power of X-rays also enables their use for non-vacuum environments such as solid-liquid interfaces. The strong X-ray beams from a synchrotron radiation source are required for these experiments. Experiments are difficult, because the surface signal is about a million times less than that from the bulk and the signal needs to be detected against a background coming from the liquid and other sources. For this reason, only highlyordered crystals with atomically flat surfaces can be investigated. The system to be studied has to be selected with this in mind.

Over the last years we have studied the solid-liquid interface structure of potassium-dihydrogen-phosphate $\left(\mathrm{KH}_{2} \mathrm{PO}_{4}, \mathrm{KDP}\right)$ with increasing levels of detail. The growth morphology of this crystal, that is grown from aqueous solution, is determined by two facets: $\{101\}$ and $\{100\}$. After determining the surface termination and relaxations, ${ }^{31}$ we also observed the ordering in the interfacial liquid. ${ }^{32}$ As an illustration of the current state-of-the-art for a crystal growth system, we present here our recent results on the $\mathrm{pH}$ dependence of the interface structure. From macroscopic observations, the growth velocity of the $\{101\}$ face is known to exhibit quite a strong dependence on the $\mathrm{pH}$ value, with a maximum for a stoichiometric solution $(\mathrm{pH}=4.4)$, while there is little $\mathrm{pH}$ dependence for the $\{100\}$ face. $^{33,34}$

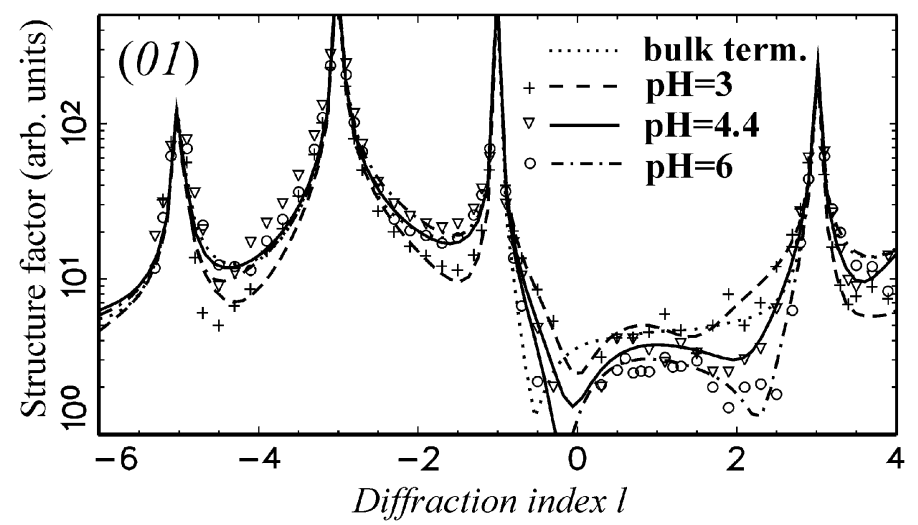

Fig. 7 The (01) crystal truncation rod of the $\operatorname{KDP}\{101\}$ surface as measured using in situ $\mathrm{X}$-ray diffraction. The structure factor amplitude is plotted versus the diffraction index $l$. The high values for $l=-5,-3,-1$ and 3 correspond to bulk reflections. In between these, the data have the highest surface sensitivity. Data (symbols) are shown for three $\mathrm{pH}$ values. The curves correspond to the best fits using a model including crystal surface relaxations and ordering in the interfacial liquid. The dotted curve is a calculation for a bulk terminated surface. The differences between the various curves are small, but significant. 
Using the DUBBLE beam line at the European Synchrotron Radiatidiefarilitye Online (ESRF), we obtained extensive data sets in the form of so-called crystal truncation rods. ${ }^{35}$ These consist of diffuse intensity connecting the bulk Bragg peaks in the direction perpendicular to the surface. Fig. 7 shows one such rod for the $\{101\}$ face measured for $\mathrm{pH}$ values of 3, 4.4 and 6 . The surface/interface sensitivity is highest (and the intensity weakest) in between the bulk Bragg peaks and the figure shows that indeed in these regions there is a significant difference between the three conditions. We are thus able to detect changes in the interfacial structure as a function of $\mathrm{pH}$. A fit of the full data set requires a model describing both relaxations in the top most crystal layers and ordering in the first few liquid layers. The fit results are shown in Fig. 7 as well. Such a fit is very reliable for the highly ordered crystalline side of the interface; for the $\{101\}$ face we find that the surface terminates in a $\mathrm{K}^{+}$
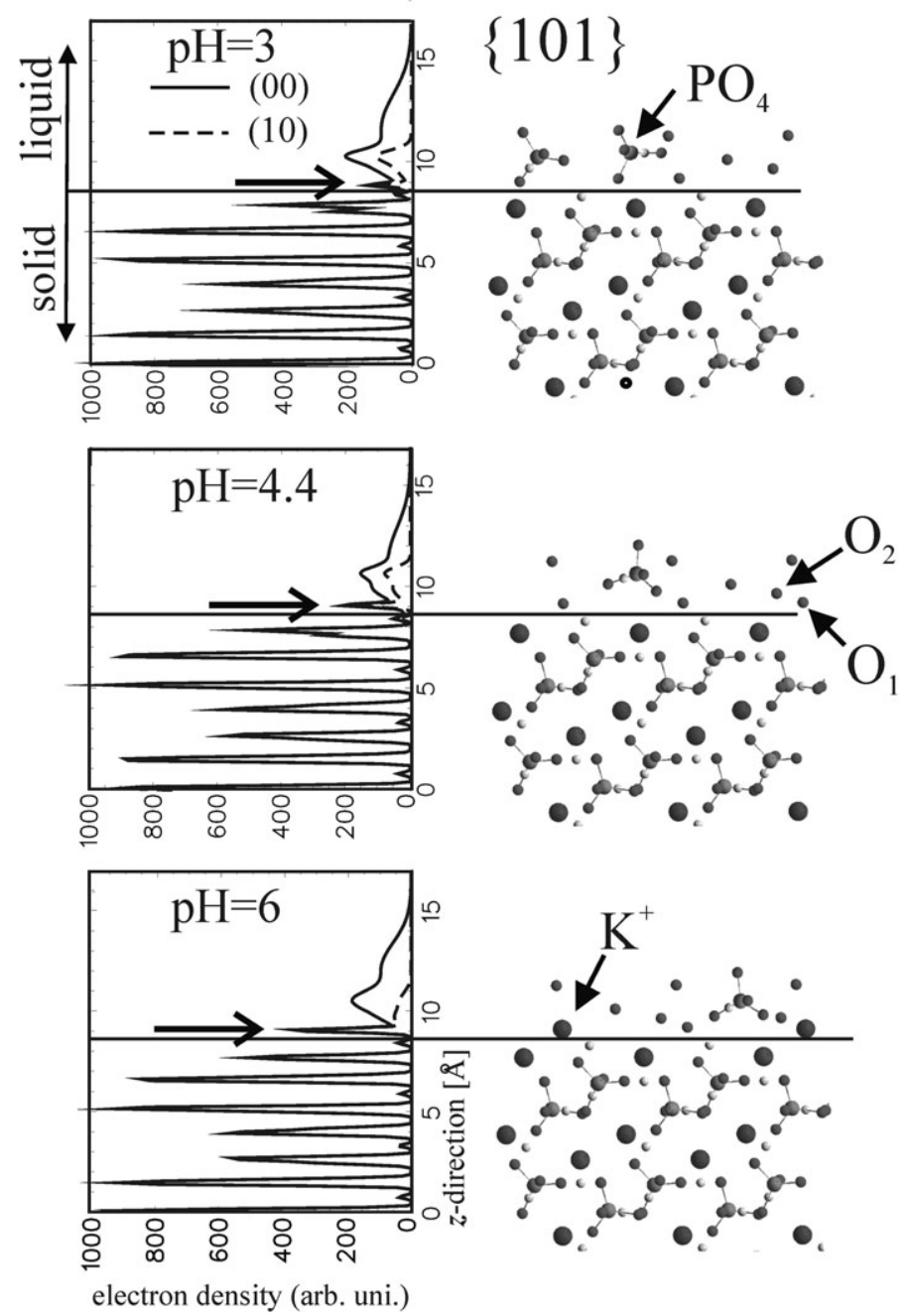

Fig. 8 The interface structure of $\operatorname{KDP}\{101\}$ for three $\mathrm{pH}$ values. On the left the projected electron density is shown, which depends on the specific Fourier component considered. The specular or (00) rod shows the full density, the (10) rod only the density with significant in-plane crystalline order. The largest variation in the experiment is indicated by the arrows. On the right a scheme of the actual interface structure is shown. For decreasing $\mathrm{pH}$ value (going from bottom to top), $\mathrm{K}^{+}$ions are more-and-more replaced by $\mathrm{H}_{3} \mathrm{O}^{+}$ions. 
layer with a small relaxation of $0.10,0.06$ and $-0.04 \AA$ for the $\mathrm{pH}$ equal toVequ. Aricte Online 6 , respectively. The relaxation in the $\mathrm{PO}_{4}{ }^{3-}$ layer directly underneath is even smaller. The structure in the liquid is more difficult to determine, because it contains $\mathrm{K}^{+}$, $\mathrm{PO}_{4} \mathrm{H}_{2}{ }^{-}$and $\mathrm{H}_{2} \mathrm{O}$ groups and is less ordered. This makes a unique assignment of atomic positions difficult. Nevertheless, when a large data set of sufficient quality is obtained, structural trends can be derived. A convenient way to show this is in the form of the calculated electron density projected on the surface normal, see Fig. 8. The amount of electron density that is visible for a particular diffraction rod depends on the parallel momentum transfer. ${ }^{32}$ For the specular rod there is no parallel momentum transfer and all perpendicular order (layering) in the liquid is visible. Other rods only probe layers that exhibit lateral order. We find approximately three ordered layers in the liquid. The most obvious change as a function of $\mathrm{pH}$ in the projected density is that the density in the first liquid layer is strongly decreasing for decreasing $\mathrm{pH}$ (indicated by arrows in Fig. 8). From a full analysis, we conclude that at $\mathrm{pH}=6$ a partial $\mathrm{K}^{+}$layer, with the ion essentially at a bulk-extrapolated position, is responsible for this high density. In the bulk of the crystal the binding is such that a positive ion is favoured at this location and this apparently remains true at the solid-liquid interface. We attribute the decrease in density to an increasing replacement of $\mathrm{K}^{+}$by $\mathrm{H}_{3} \mathrm{O}^{+}$, because the concentration of the latter increases (by definition) by a factor 1000 . Its concentration remains low with respect to that of $\mathrm{K}^{+}$, but the possibility to form additional $\mathrm{H}$-bonds near the interface makes $\mathrm{H}_{3} \mathrm{O}^{+}$ more favourable. $^{36}$

A similar analysis for the $\{100\}$ face shows no significant changes in the XRD data as a function of $\mathrm{pH}$ and thus the interface structure remains constant. The main feature we derive from the analysis is an $\mathrm{H}$-bond between an oxygen from the topmost $\mathrm{PO}_{4}{ }^{3-}$ layer and a water molecule, see Fig. 9. This compensates for a broken $\mathrm{H}$-bond due to the crystal termination. This new bond will not be affected by the change in $\mathrm{pH}$, which explains the insensitivity of the $\{100\}$ face to the $\mathrm{pH}$ value. The chemisorbed water molecule is found to be well-ordered. ${ }^{37}$

The structural changes at the $\{101\}$ face and the absence of such changes at the $\{100\}$ face as a function of $\mathrm{pH}$ correlate with the fact that the corresponding changes in the macroscopic growth velocity are large for the $\{101\}$ and small for the $\{100\}$ face. Unfortunately, we cannot directly translate the structural changes at the $\{101\}$ face into changes in the expected growth velocity. There appears to be a monotonous change in $\mathrm{K}^{+}$versus $\mathrm{H}_{3} \mathrm{O}^{+}$bonding, but the maximum growth velocity is found for

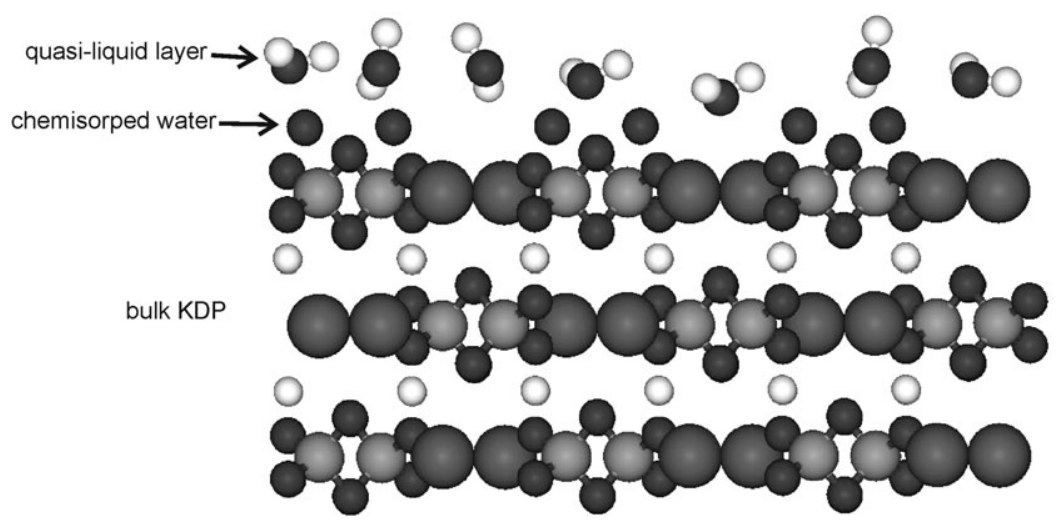

Fig. 9 Side view of the KDP(100) surface, showing the chemisorbed water molecules at the interface that compensates the broken hydrogen bonds due to the surface termination. The water molecule is represented by a single oxygen atom, because X-rays are very insensitive to hydrogen atoms. The data analysis also shows order in the form of layering in the second water layer. 
the middle $\mathrm{pH}$ value of 4.4. The actual growth for both faces involves severialwitreitite Online barriers and these will depend on the species present at the interface.

\section{Closing the gap?}

When comparing the two recent results presented above in morphology prediction and on the interface structure of KDP, it is clear that there is a large gap in the level of detail included in the two methods. The morphology prediction assumes a bulkterminated crystal, without surface relaxations and it ignores the solution and possible effects of chemisorption. The main variable in the experimental results on $\mathrm{KDP}$, the $\mathrm{pH}$ value of the solution, is not a parameter in the theory. It is by no means obvious how to include such effects. Interaction with the solution will generally change the stability of steps and the change in energy will depend on the step structure. If a method to estimate such interactions was available, this could be included in the step energy method and a modified morphology could be calculated.

It is our belief that the development of more sophisticated growth theories needs the inspiration obtained from computer simulations. In fact, the step energy method was developed after performing Monte Carlo simulations using the Monty program $^{38}$ that uses the same crystal graph, but also includes the effects of kinks and step entropy. Such simulations showed that the step energy is the dominant parameter and thus support our theory that ignores the less important effects.

Understanding the effects of surface relaxations or solvent interactions requires simulations beyond a lattice model. This is slowly happening along two directions. On the one hand first-principle calculations can help in understanding the equilibrium solid-liquid structure. Such calculations have recently been performed for $\mathrm{KDP}^{7}$ and were found to be in promising agreement with the experimental results. The other approach is through Molecular Dynamics simulations using effective potentials to describe the interactions between the various components in the growth system. The growth interface of KDP is still beyond the reach of current methods, but for a limited number of systems involving less components, encouraging results have been obtained. ${ }^{6,39-41}$ These realistic calculations are very costly and the system size has to be quite small. Nevertheless, in this area a genuine contact between experiment and modelling is emerging.

We hope and expect that progress will continue to be made in both first-principle calculations and in MD simulations. This will help in understanding growth kinetics and will guide the development of more detailed growth theories. In this way the overlap between theory/modelling and experiment will increase together with our understanding of crystal growth at the atomic scale.

\section{Acknowledgements}

We thank the DUBBLE staff for assistance during the XRD experiments. This work was made possible by financial support from Synthon B. V. and the Council for Chemical Sciences of the Netherlands Organisation for Scientific Research (CW-NWO).

\section{References}

1 W. K. Burton, N. Cabrera and F. C. Frank, Philos. Trans. R. Soc. London, Ser. A, 1951, 243, 299-358.

2 G. H. Gilmer and P. Bennema, J. Appl. Phys., 1972, 43, 1347-1360.

3 H. M. Cuppen, H. Meekes, W. J. P. van Enckevort, E. Vlieg and H. J. F. Knops, Phys. Rev. B, 2004, 69, 245-404.

4 A. A. Chernov, J. Mater. Sci., 2001, 12, 437-449.

5 H. M. Cuppen, H. Meekes, W. J. P. van Enckevort and E. Vlieg, Surf. Sci., 2004, 571, 41-62.

6 X. Y. Liu, E. S. Boek, W. J. Briels and P. Bennema, Nature, 1995, 374, 342-345.

7 D. J. Carter, A. L. Rohl and J. D. Gale, J. Chem. Theor. Comput., 2006, 2, 797-800. 
8 H. E. Buckley, Crystal growth, Wiley, New York, 1951.

9 J. Nyvlt and J. Ulrich, Admixtures in crystallization, VCH, Weinheim, 1995.

10 G. Sazaki, K. Tsukamoto, S. Yai, M. Okada and K. Nakajima, Cryst. Growth Des., 2005, 5, 1729-1735.

11 G. Wulff, Z. Krist. Mineral., 1901, 34, 449.

12 A. Bravais, Etudes Cristallographiques, Paris, 1866.

13 G. Friedel, Bull. Soc. Fr. Mineral., 1907, 30, 326.

14 J. D. H. Donney and G. Harker, Am. Mineral., 1937, 22, 446-467.

15 P. Hartman and W. G. Perdok, Acta Crystallogr., 1955, 8, 49-52.

16 P. Bennema, in Handbook of Crystal Growth, ed. D. T. J. Hurle, North-Holland, Amsterdam, 1993, vol. 1B, pp. 477-582.

17 P. Hartman and P. Bennema, J. Cryst. Growth, 1980, 49, 145-156.

18 R. F. P. Grimbergen, H. Meekes, P. Bennema, C. S. Strom and L. J. P. Vogels, Acta Crystallogr., 1998, A54, 491-500.

19 M. A. Deij, J. Los, H. Meekes and E. Vlieg, J. Appl. Crystallogr., 2006, 39, 563-570.

20 N. Akutsu and Y. Akutsu, J. Phys. Soc. Jpn., 1995, 64, 736-756.

21 H. van Beijeren and I. Nolden, in Structure and Dynamics of Surfaces II, ed. W. Schommers and P. van Blanckenhagen, Springer, Berlin, 1987, pp. 259-300.

22 M. A. Deij, H. Meekes and E. Vlieg, Cryst. Growth Des., submitted.

23 J. P. van der Eerden, in Handbook of Crystal Growth, ed. D. T. J. Hurle, Elsevier Science Publisher, Amsterdam, 1993, vol. 1, pp. 307-475.

24 M. A. Deij, J. van Eupen, H. Meekes, P. Bennema and E. Vlieg, to be published.

25 H. M. Cuppen, G. Beurskens, S. Kozuka, K. Tsukamoto, J. M. M. Smits, R. de Gelder, R. F. P. Grimbergen and H. Meekes, Cryst. Growth Des., 2005, 5, 917-923.

26 T. A. Land, T. L. Martin, S. Potapenko, G. T. Palmore and J. J. De Yoreo, Nature, 1999, 399, 442-445.

27 M. Plomp, W. J. P. van Enckevort and E. Vlieg, J. Cryst. Growth, 2000, 216, 413-427.

28 E. Vlieg, Surf. Sci., 2002, 500, 458-474.

29 R. Feidenhans'l, Surf. Sci. Rep., 1989, 10, 105-188.

30 I. K. Robinson, in Handbook on Synchrotron Radiation, ed. G. S. Brown and D. E. Moncton, North-Holland, Amsterdam, 1991, vol. 3, pp. 221-266.

31 S. A. de Vries, P. Goedtkindt, S. L. Bennett, W. J. Huisman, M. J. Zwanenburg, D.-M. Smilgies, J. J. De Yoreo, W. J. P. van Enckevort, P. Bennema and E. Vlieg, Phys. Rev. Lett., 1998, 80, 2229-2232.

32 M. F. Reedijk, J. Arsic, F. F. A. Hollander, S. A. de Vries and E. Vlieg, Phys. Rev. Lett., 2003, 90, 066103.

33 L. N. Rashkovich and G. T. Moldazhanova, J. Cryst. Growth, 1995, 151, 145-152.

34 S. K. Sharma, S. Verma, B. B. Shrivastava and V. K. Wadhawan, J. Cryst. Growth, 2002, 244, 342-348.

35 I. K. Robinson, Phys. Rev. B, 1986, 33, 3830-3836.

36 D. Kaminksi, N. Radenovic, M. A. Deij, W. J. P. van Enckevort and E. Vlieg, Phys. Rev. $B, 2005,72,245404$.

37 D. Kaminksi, N. Radenovic, M. A. Deij, W. J. P. van Enckevort and E. Vlieg, Cryst. Growth Des., 2006, 6, 588-591.

38 S. X. M. Boerrigter, G. P. H. Josten, J. van de Streek, F. F. A. Hollander, J. Los, H. M. Cuppen, P. Bennema and H. Meekes, J. Phys. Chem. A, 2004, 108, 5894-5902.

39 M. Brunsteiner and S. L. Price, J. Phys. Chem. B, 2004, 108, 12537-12546.

40 C. Stoica, P. Verwer, H. Meekes, P. J. C. M. van Hoof, F. M. Kaspersen and E. Vlieg, Cryst. Growth Des., 2004, 4, 765-768.

41 S. Piana, M. Reyhani and J. D. Gale, Nature, 2005, 438, 70-73. 